Los, Gerrit

[45]

\section{Unique expression patterns for paclitaxel, doxorubicin and cisplatin in breast carcinoma cells}

Fei Yang, Gerald Manorek \& Gerrit Los

University of California San Diego Cancer Center, La Jolla, California, USA

Differences in mechanisms of action between paclitaxel, doxorubicin and cisplatin in breast cancer may be accompanied by a corresponding diversity in gene expression patterns. We analyzed gene expression patterns in the MCF-7 cell line at different times $(6,24,48$ and $72 \mathrm{~h})$ after treatment with paclitaxel, doxorubicin or cisplatin, using complementary microarrays representing 1,308 human complementary DNAs. The microarrays were created from six suppression subtractive hybridization libraries, representing complementary DNAs to messenger RNAs that were either upor downregulated in MCF-7 cells after treatment with either paclitaxel, doxorubicin or cisplatin $\left(\mathrm{IC}_{90}\right.$ concentrations). We used a hierarchical clustering method to group genes on the basis of similarity between expression patterns in each treatment. Two striking aspects of the patterns were evident. First, the expression pattern of each drug was unique for that drug. Fewer than $2 \%$ of the 1,308 human cDNAs present on the microarray were differentially expressed for all three drugs. Second, within each unique drug expression pattern, distinct clusters could be detected. It was clear from time-course hybridizations that clusters could be divided into early-, intermediateand late-response genes. Only a small percentage of the fragments are common to the cellular injury produced by any of these drugs. The majority of the changes in mRNA expression observed with any one drug were unique to that drug, providing the foundation for a clinically applicable technology to develop a breast cancer response chip.

Lucito, Robert [46]

\section{Detection of gene copy number fluctuations with microarrays using genomic representations}

Robert Lucito, Joan Alexander, Seth Rostan, Joseph West $\&$ Michael Wigler

Cold Spring Harbor Laboratory, Cold Spring Harbor, New York, USA

We have developed a microarray method, based on genomic representations, designed to measure genome copy number fluctuations. A representation is a reproducible sampling of the genome, prepared by digesting genomic DNA with a restriction enzyme such as BglII, ligation of adaptors and polymerase chain reaction amplification. The representation is reduced in complexity in comparison with the original genomic DNA, resulting in an increase in the ratio of signal to noise on the microarray. The probes on the microarray are fragments cloned from a representation of a normal male, and the samples hybridized are representations of genomic DNA made in the same manner as the arrayed fragments. We had previously shown that representations of genomic DNA were faithful and conserved copy number between a test and a reference sample. We now show that representations can be used in a microarray format for analysis on a genome-wide scale, and that this method is reproducible and quantitative. We have used this method to analyze the genome copy number fluctuations in primary breast cancer. We separated specimens into tumor and normal samples by ploidy, prepared representations, labeled them and hybridized them to microarrays. The results were analyzed to identify regions that have undergone gene copy number fluctuations. We have assessed the frequency with which various regions, such as erbB2, have been affected. We have also used this method in an attempt to identify uncharacterized regions. These regions will be subject to closer scrutiny, to identify genes that may play a role in the promotion of primary breast tumorigenesis.
Lushnikova, Tamara

[47]

\section{DNA copy number changes in epithelioid sarcoma and its variants}

\author{
Tamara Lushnikova ${ }^{1,2}$, Knuutil Sakari ${ }^{3} \&$ Miettinen Markku ${ }^{4}$ \\ ${ }^{1}$ University of Tartu, Estonia \\ ${ }^{2}$ Marshall University, Huntington, West Virginia USA ${ }^{3}$ Haartman Institute, \\ University of Helsinki, Finland ${ }^{4}$ Department of Defense, Armed Forces Institute \\ of Pathology, Washington DC, USA
}

Epithelioid sarcoma is a distinctive, relatively rare soft tissue sarcoma that typically involves the distal extremities in young adults and shows immunohistochemical markers of epithelial differentiation. The genetic background of epithelioid sarcoma is poorly understood, and knowledge of it could give insights into the pathogenesis of this tumor and its possible relationship with other malignant tumors. In this study, we analyzed DNA copy number changes in 30 epithelioid sarcomas by comparative genomic hybridization. DNA was extracted from microdissected samples of formaldehyde-fixed and paraffin-embedded tumors with a mininum of $60 \%$ of tumor cells in each sample. Sixteen tumors (53\%) showed DNA copy number changes at 1-7 different genomic sites. The majority of the changes were gains, seen in 15 tumors, whereas 12 tumors showed losses. We detected recurrent gains at $11 \mathrm{q} 13$ ( 5 cases), 1q21-q23 (4 cases), 6p21.3 (3 cases), 9q31-qter ( 3 cases). Highlevel amplifications were detected once in 6p21.3-p21.1 and once in 9q32-qter. Recurrent losses were seen at 9p21 (3 cases), 13q22-q32 (3 cases), 1p13-p22 (2 cases), 3p12-p14 ( 2 cases), 4q13-q33 ( 2 cases), 9pter-p23 (2 cases) and 13q32-qter ( 2 cases). The most common recurrent gain at $11 \mathrm{q} 13$ was also seen in both classical cases and angiomatoid and rhabdoid variants, supporting the relationship of these variants with the classical epithelioid sarcoma. The observed changes give targets for future genetic studies on epithelioid sarcoma.

Madden, Stephen

[48]

\section{Transcriptome annotation of genomes using SAGE}

Stephen Madden, Brian Cook, Mindy Zhang, Clarence Wang $\&$ Katherine Klinger

Genzyme Molecular Oncology, Framingham, Massachusetts 01701, USA

Wordwide use of the Serial Analysis of Gene Expression (SAGE) database has resulted in a vast amount of gene expression information. This immortal database couples quantitative transcript abundance data to specific genes and allows for the defining and delineation of new, active transcription units. Recent advances in SAGE technology have allowed the unambiguous, direct linking of SAGE tag data to complex, whole-genome data. With a vast, cancer-specific SAGE database in hand, researchers now have an opportunity for comprehensive, genome-wide transcriptome annotation relating to cancer. 